\title{
Quantum dot photonic-crystal-slab nanocavities: Quality factors and lasing
}

\author{
J. Hendrickson, B. C. Richards, J. Sweet, S. Mosor, C. Christenson, D. Lam, G. Khitrova, and H. M. Gibbs \\ Optical Sciences Center, The University of Arizona, Tucson, Arizona 85721, USA \\ T. Yoshie \\ Department of Electrical and Computer Engineering, Duke University, Durham, North Carolina 27708, USA \\ A. Scherer \\ Department of Electrical Engineering, California Institute of Technology, Pasadena, California 91125, USA \\ O. B. Shchekin and D. G. Deppe \\ Microelectronics Research Center, Department of Electrical and Computer Engineering, The University of Texas at Austin, \\ Austin, Texas 78712, USA \\ (Received 22 August 2005; published 9 November 2005)
}

\begin{abstract}
Emission linewidths of quantum dot photonic-crystal-slab nanocavities are measured as a function of temperature and fabrication parameters with low-power and high-power, cw and pulsed, nonresonant excitation. The cavity linewidth is dominated by the absorption of the ensemble of quantum dots having a density of $\cong 400 / \mu \mathrm{m}^{2}$; above the absorption edge, the cavity linewidth broadens considerably compared with the empty cavity linewidth. Gain and lasing are seen for high-power pumping; it is estimated that only a small number of quantum dots contributes to the lasing.
\end{abstract}

DOI: $10.1103 /$ PhysRevB.72.193303

PACS number(s): 42.55.Tv, 42.50.Pq, 42.55.Sa

Recently the quality factor $Q$ [mode energy divided by full width at half maximum (FWHM) mode energy linewidth] of photonic-crystal-slab cavities has been steadily increased by improved fabrication techniques and designs, while the volume $V$ was kept close to a cubic wavelength in the material. This has made possible not only quantum well ${ }^{1}$ and quantum $\operatorname{dot}^{2}$ lasers but also the observation of strong coupling ${ }^{3}$ - vacuum Rabi splitting with a single quantum dot (SQD). The role of the quantum dots (QDs) in the lasers is to provide gain, so several layers of high density QDs are often used. In contrast, strong coupling, can best be observed with an isolated SQD, suggesting the use of a single layer of low density QDs. However, to see strong coupling one must search to find two accidental coincidences. The QD must be situated close to an intracavity field maximum. This means it must be within the mode area of $0.15 \mu \mathrm{m}^{2}$, where the intracavity field is strong. It must also have a transition frequency close to a cavity mode; our ensemble QD lowest energy transition has a FWHM of $42.5 \mathrm{meV}$ at $20 \mathrm{~K}$, compared with a maximum dot-nanocavity coupling strength of $0.2 \mathrm{meV}$. For a reasonable probability for both coincidences, high dot densities $\left(300-400 / \mu \mathrm{m}^{2}\right)$ have been used so far. This paper addresses two questions: Is the ensemble QD absorption detrimental to the search for strong coupling? And, if the gain is sufficient for lasing, roughly how many QDs contribute?

To fabricate a photonic-crystal-slab nanocavity, a sample is grown by molecular beam epitaxy on a (001) GaAs substrate starting with a GaAs buffer layer: $800 \mathrm{~nm}$ $\mathrm{Al}_{0.94} \mathrm{Ga}_{0.06} \mathrm{As}$ sacrificial layer, $40 \mathrm{~nm}$ GaAs, $20 \mathrm{~nm}$ $\mathrm{Al}_{0.1} \mathrm{Ga}_{0.9} \mathrm{As}$, single layer of self-assembled InAs QDs (density of $300-400 \mu \mathrm{m}^{2}$ ), and on top of the dots $20 \mathrm{~nm}$ $\mathrm{Al}_{0.1} \mathrm{Ga}_{0.9} \mathrm{As}$ and $40 \mathrm{~nm} \mathrm{GaAs} .{ }^{4}$ Then a two-dimensional triangular photonic-crystal-lattice with three holes missing to form a cavity spacer is fabricated to provide in-plane light confinement. The GaAs-air interfaces on the top and bottom of the 270-nm-thick slab provide vertical confinement by means of total internal reflection, but light with small inplane wave vectors still leaks out of the cavity. As shown by Noda's group using $\mathrm{Si}$, vertical confinement is further enhanced by slightly shifting outward the holes at the ends of the spacer; the $Q$ is increased by confining gently. ${ }^{5}$

The quantum dots are excited by a continuous-wave Ti:Sapphire laser (wavelength $780 \mathrm{~nm}$ ) or alternatively by a $20 \mathrm{~ns}$ pulsed diode laser (wavelength $784 \mathrm{~nm}$, Sharp Microelectronics, $2.5 \%$ duty factor). A 0.5 numerical aperture (NA) microscope objective is used to focus the beam to a spot size of 1 to $2 \mu \mathrm{m}$ diameter. The sample is mounted on nanopositioners inside a continuous flow liquid-helium cryostat. The photoluminescence (PL) is collected in reflection geometry back through the same microscope objective, imaged with $f$-number matching into a spectrometer, and detected by an InGaAs photodiode linear array.

Figure 1(a) shows that the ensemble QD PL spectra at 20 and $200 \mathrm{~K}$ are almost the same, except band gap shrinkage shifts the PL peak from 1.020 to $0.982 \mathrm{eV}$. Because of the very large inhomogeneous linewidth due to the size distribution, the absorption and PL spectra are not expected to change much with temperature.

The idea behind Figs. 1(b) and 1(c) is to determine the effect of absorption and gain on the cavity $Q$ by measuring the nanocavity mode linewidth as a function of temperature [Fig. 1(b)] or of fabrication parameters [Figs. 1(b) and 1(c)]. As the temperature is increased from 20 to $200 \mathrm{~K}$, the cavity peak $E_{c a v}$ shifts to lower energy by $8.2 \mathrm{meV}$ compared with the much larger $38 \mathrm{meV}$ shift of the QD ground state peak $E_{0}$. Therefore, temperature scans the detuning, $E_{c a v}-E_{0}$, permitting a portion of the linewidth versus detuning curve to be determined. By using five nanocavities, labeled 1 to 5 in Fig. 

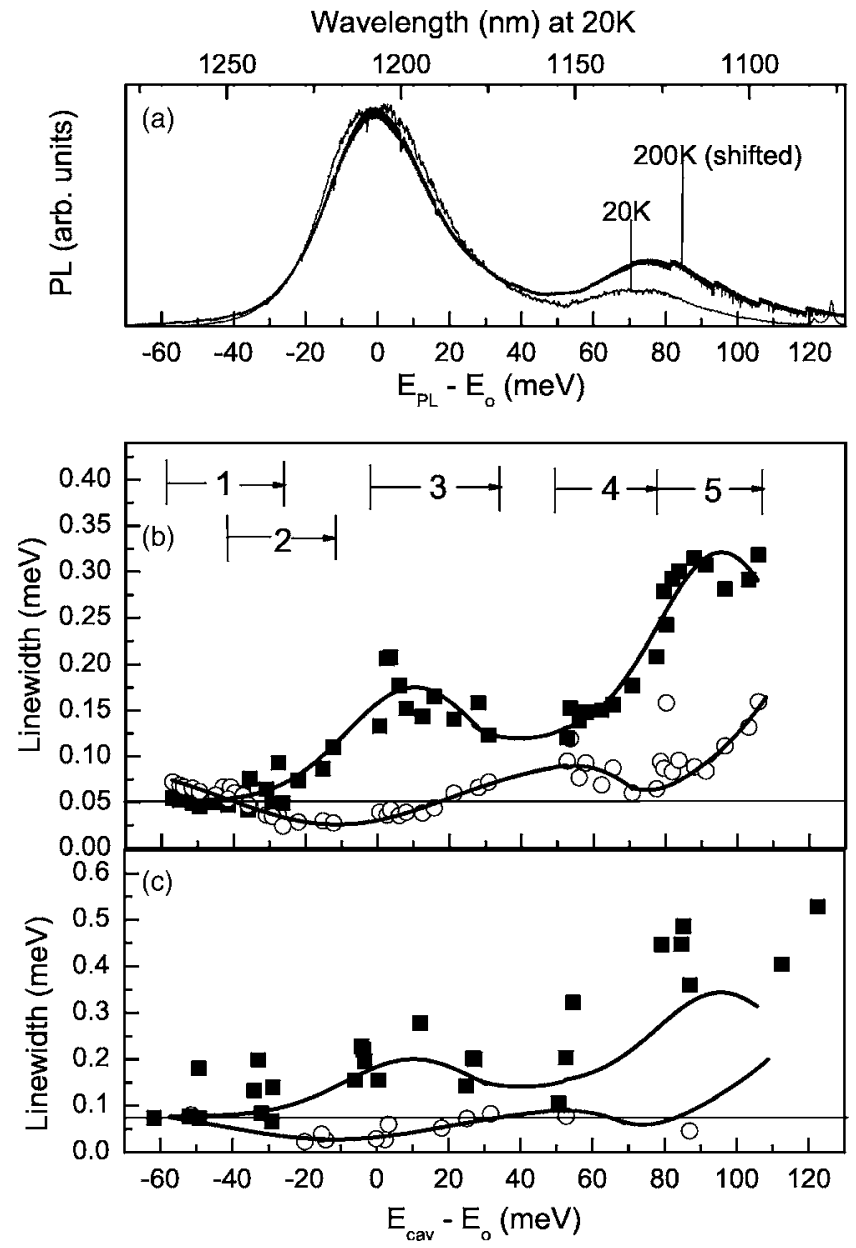

FIG. 1. Quantum dot emission spectra and cavity linewidths. (a) Ensemble QD emission spectra at 20 and $200 \mathrm{~K}$ versus energy relative to the ensemble QD ground-state transition peak $\mathrm{E}_{0}$. (b) Nanocavity FWHM linewidth for several temperatures between 20 and $200 \mathrm{~K}$ versus the detuning of the nanocavity mode peak from the ensemble QD ground-state transition peak. The solid squares are for low-power $(2-10 \mu \mathrm{W}) \mathrm{cw}$ excitation, and the open circles are for high-power $(720 \mu \mathrm{W}) \mathrm{cw}$. Five nanocavities were selected that cover the energies of the QD ground-state and first-excited-state transitions, with the detuning increasing with temperature. All five have a $r / a=0.27$ and $s / a=0.20$ and were from the same fabrication run. The data points and guide-to-the-eye solid curves show that the low-power linewidths follow the expected ensemble QD absorption spectrum. But the high-power linewidth drops below the emptycavity straight line, indicating the presence of gain. (c) Nanocavity FWHM linewidth for low temperatures around $20 \mathrm{~K}$ versus detuning. The nanocavities were selected from two different fabrication runs and have a wider range of lattice constants, radii, shifts, and dosing values than those in (b). Solid squares: 2-20 $\mu \mathrm{W} \mathrm{cw}$; open circles: $1 \mathrm{~mW}$ diode-laser peak power. The solid curves are the same ones in (b) with a slight shift upward to take account of the higher average empty-cavity linewidth. The behavior of the linewidth is clearly similar to that in (b).

1(b), and choosing different fabrication parameters such as $e$-beam dosing, hole radius $r$, lattice constant $a$, and shift $s$ of "spacer" end holes (but always keeping $r / a=0.27$ and $s / a$ $=0.20$ ), the ground-state and first-excited-state spectral re- gion was covered. The top set of data and guide-to-the-eye curve in Fig. 1(b) show that the low-power linewidth spectrum has the shape expected for the QD ensemble absorption with peaks at both the ground-state and first-excited-state absorption transitions. This is essentially an intracavity measurement of the absorption's spectral profile. Both ensemble ${ }^{6}$ and single ${ }^{7}$ quantum dot absorptions have been measured previously. The absorption spectrum extracted from our linewidth data is much like that obtained from the photocurrent in a waveguide containing InAs QDs (see Fig. 1 in Ref. 8). They do not find a shift between PL and absorption peaks, and we doubt that the apparent shift in our Fig. 1 is statistically significant.

One can also keep the temperature fixed at $20 \mathrm{~K}$ and use the fact that the nanocavity wavelength depends upon fabrication parameters; see the upper data points in Fig. 1(c). It is not surprising that this more random selection of nanocavities has greater variations in $\delta E_{\text {empty }}$, but the behavior is the same as Fig. 1(b). Clearly the QD ensemble absorption controls the linewidth $\delta E$ (and therefore $Q=E / \delta E$ of our nanocavities, except at the low-energy tail of the distribution where the absorption is small. For these data, the input power was kept low enough that saturation ${ }^{9}$ of the QD absorption was negligible. Uncoupled or poorly coupled QDs have longer radiative lifetimes than QDs with Purcell enhancement or strong coupling, therefore, they can be saturated by pumping hard enough-their emission rate becomes limited by their longer lifetimes. Note that the linewidths and $Q$ s were the same for QD excited-state pumping as for pumping into the GaAs.

The empty cavity linewidth, determined from the large negative detuning points in Fig. 1(b) for which absorption is expected to be negligible, is about $0.05 \mathrm{meV}$ (corresponding to $\left.Q_{\text {empty }}=20000\right)$. One expects $1 / Q_{\text {empty }}=1 / Q_{\text {design }}$ $+1 / Q_{\text {growthlfab }} ; Q_{\text {design }}$ (computed to be $40000-50000$ here) accounts for design losses that are present even if the fabrication is perfect, whereas $Q_{\text {growth/fab }}$ comes from additional scattering losses due to fluctuations introduced by growth and etching. Since neither $Q_{\text {design }}$ or $Q_{\text {growthlfab }}$ is expected to vary much over this wavelength range, a straight line is drawn all the way across Fig. 1(b) at $0.05 \mathrm{meV}$ to indicate $\delta E_{\text {empty. }}$.

How do these findings affect the search for QD nanocavities that exhibit strong coupling? Since the dot-nanocavity coupling $g$ must exceed the cavity loss rate $\kappa=2 \pi v / Q$, it is $g / \kappa \propto Q / \sqrt{V}$ that needs to be maximized for strong coupling. We find that at the low powers required for strong coupling, the QD ensemble absorption is large enough to reduce the $Q$ by at least a factor of 2 for QD transitions near the top of the ensemble distribution or above it. From this perspective, cavities near the low-energy tail of the QD spectrum should present a better opportunity for the observation of strong coupling due to much lower QD absorption and higher Qs. However, the low absorption corresponds to a lower density of QDs, meaning that for a particular cavity, there is a lower likelihood of finding a QD which has the spatial and spectral coincidences necessary for strong coupling. Temperature scanning can change the dot-nanocavity detuning by about $0.7 \mathrm{~nm}$ before phonon broadening prevents strong coupling. Recently, we have shown that condensation of nitrogen or 
xenon can give much larger shifts $(\sim 4 \mathrm{~nm})$, making it easier to find the required coincidences using lower QD densities. ${ }^{10}$ In another approach (that did not achieve strong coupling), a photonic crystal nanocavity was fabricated around a particular QD, and etching was used to scan the nanocavity peak digitally in $3 \mathrm{~nm}$ steps. ${ }^{11}$

Above, we have studied the cavity linewidth and $Q$ for low-power excitation appropriate for strong coupling and showed that indeed the ensemble absorption of a single layer of high-density QDs is detrimental. A related investigation can be performed to determine if the single layer of QDs can produce gain and lasing when excited sufficiently strongly. The lower set of data in Fig. 1(b) is taken with high-power cw excitation for which the electron and hole ground states of most of the QDs in the ensemble are occupied; gain is apparent over the range -30 to $+10 \mathrm{meV}$ detuning where the linewidth drops well below $\delta E_{\text {empty. }}$. Even the linewidths for QDs in the lower-energy part of the first-excited-state transition drop down almost to $\delta E_{\text {empty }}$. Furthermore, in Fig. 1(c), the lower data points for high-power diode-laser pumping show pronounced gain, similar to Fig. 1(b).

The QD nanocavity $Q$ and emission intensity as a function of the value of the peak input diode-laser power are shown in Fig. 2; a low duty factor is used to avoid heating that prevents lasing. Generally, cavities with modes lying in the upper half of the ensemble QD lowest-energy transition, where there is no gain in Figs. 1(b) and 1(c), exhibit output versus input curves like the "nonlasing" curves in Fig. 2. In this case, the output increases sublinearly and $Q$ saturates at $Q_{\text {empty }}$. This implies that the QD absorption is saturated at high powers, but there is no lasing. For cavities with modes in the lower half, where there is gain in Figs. 1(b) and 1(c), the output versus input resembles the "lasing" curves in Fig. 2 , where the output increases superlinearly, and $Q$ reaches $\cong 40000$ (determined by the spectrometer array resolution). Clearly $Q_{\text {empty }}$ is exceeded because the gain from the pumped QD ensemble overcomes some or all of the cavity losses. The case that lasing is occurring is especially strong in Fig. 3 , where the $Q$ saturates at $10 \%$ of the maximum pump power. As for the first photonic-crystal-nanocavity lasing using quantum dots, ${ }^{2,4}$ the lasing nanocavities here exhibit linewidth narrowing and a threshold behavior for output versus input. The approximate power here is $100-200 \mu \mathrm{W}$, about the same as previously. ${ }^{2,4}$ However, the absorbed power in our case is five times less, due to only one layer of dots compared with their five layers of dots.

The threshold curves are "softer" as expected for a higher $\beta$ (where $\beta$ is the rate of enhanced spontaneous emission into the lasing mode divided by the rate of total spontaneous emission ${ }^{12,13}$ ), leaving the threshold power ill-defined. For a SQD in the field maximum, the Purcell factor is $F_{p}$ $=3 \lambda^{3} Q /\left(4 \pi^{2} V\right) \cong 900$ here, provided the SQD linewidth is less than the cavity linewidth (certainly the case here for low temperature and low-excitation power). Since $\beta \cong\left(F_{p}\right.$ $-1) / F_{p}$, then $\beta \cong 1$. Of course, most QDs are not in the field anti-node and the QD linewidth may exceed the cavity linewidth at high-excitation levels, reducing the average $F_{P}$. But since the maximum $F_{P}$ is so huge, $\beta$ must be very large, i.e., between 0.1 and 1 .

In Ref. 4, it was concluded that about 80 QDs contribute to the lasing. The QDs here are identical to those and have

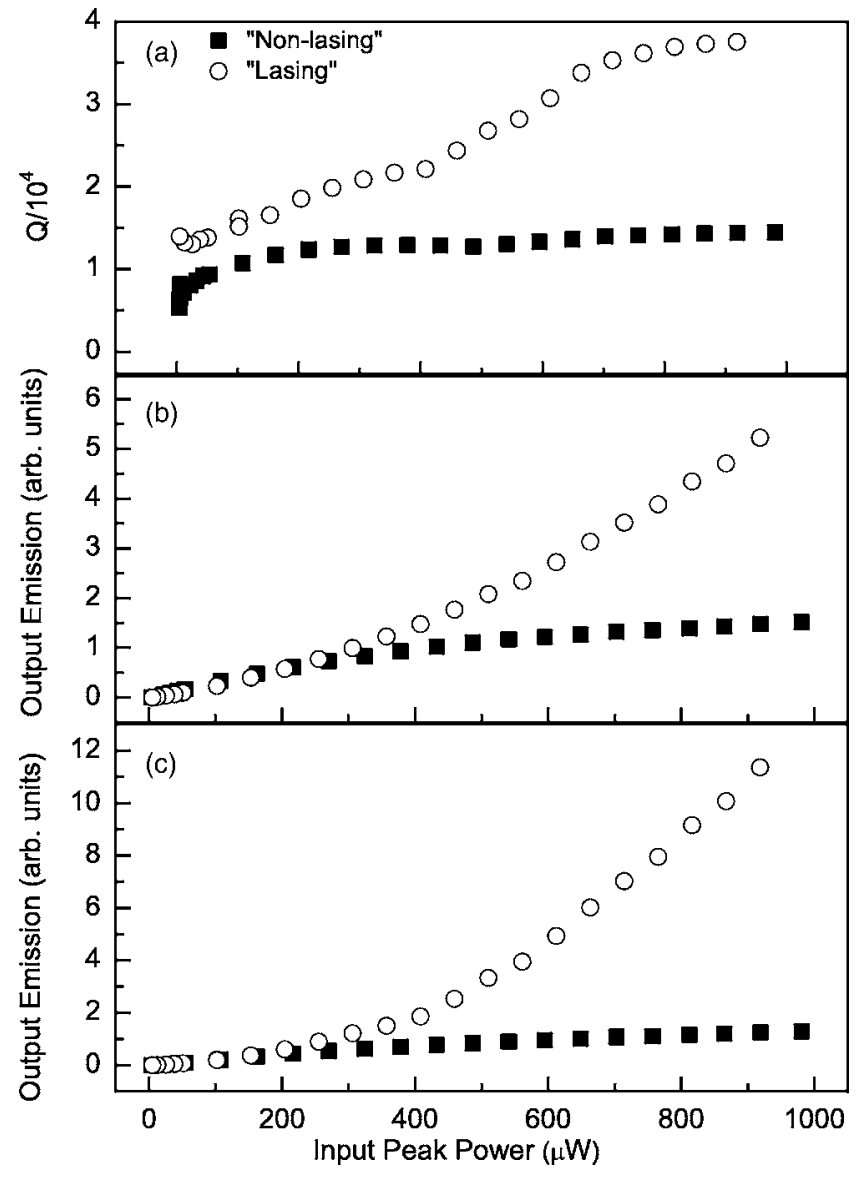

FIG. 2. Quality factor $Q$ and nanocavity output emission intensities versus diode-laser peak pump power. (a) The $Q$ of nonlasing nanocavities saturates at the empty-cavity $Q$, but the $Q$ of lasing cavities reaches the instrument-limited $Q$ of about 40000 . Correspondingly, both the time-averaged total (b) and peak (c) output emission energy exhibit a threshold-like behavior for the lasing cavities and a saturation behavior for the nonlasing cavities.

the same density in each layer, and both nanocavities have $V \cong \lambda^{3}$. However, here there is a single layer of dots compared with five layers in Ref. 4, so the number of contributing QDs is no more than 16 here. In addition, the lattice temperature here is $10-20 \mathrm{~K}$, compared with room temperature; therefore, the QD linewidth should be narrower than their $7 \mathrm{meV}$. By choosing QDs in the 1080-1092 nm spectral region, we can see individual QD transitions and measure their linewidths as a function of diode-laser peak power. For 100-200 $\mu \mathrm{W}$ peak powers (corresponding to threshold), the QD linewidth is about $0.09 \mathrm{meV}$. From Fig. 9 of Ref. 4, one then concludes that a SQD with such a linewidth should be able to lase provided the $Q$ exceeds 1000 , which is clearly the case here. Studies of lasing with an isolated SQD will be highly interesting, and the conditions here are close to those needed. ${ }^{4}$ However, SQD lasing is not claimed here, because the threshold behavior is relatively insensitive to detuning and temperature and does not change when a nanocavity is used that exhibits a strong coupling anti-crossing at low power. Most likely the gain, not from a SQD, but from several QDs in the ensemble mostly situated in the outer reaches of the cavity mode field is responsible for the lasing seen 


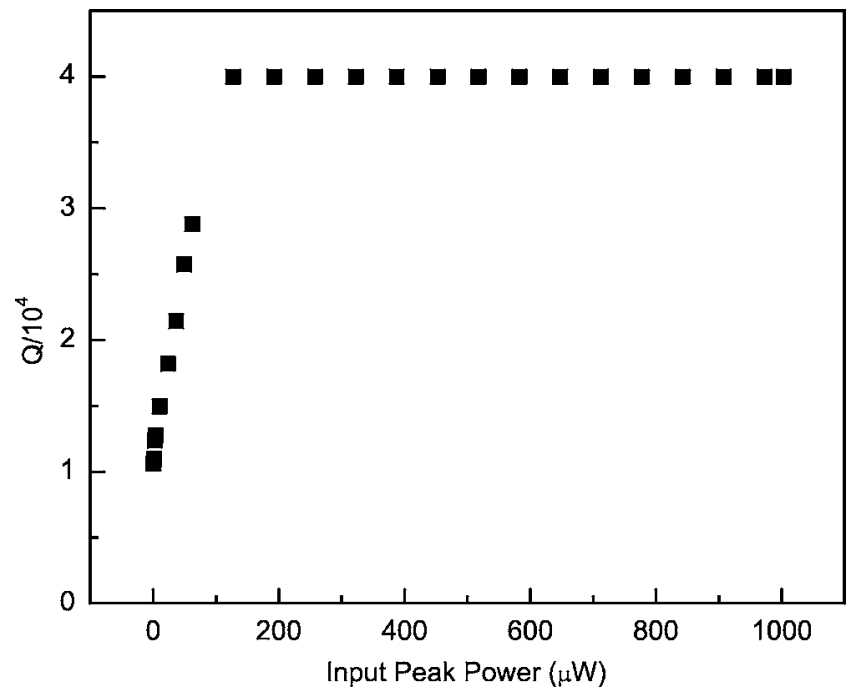

FIG. 3. Nanocavity $Q$ versus diode-laser peak pump power. The $Q$ of this nanocavity reaches the instrument-limited value of 40000 at roughly $10 \%$ of the maximum input power.

here. Additionally, phonon-assisted scattering from the dot ensemble into the cavity mode could increase the number of contributing dots. ${ }^{14}$ Clearly, a lower density of QDs in the present nanocavity would increase the chance of observing SQD lasing.

Strong coupling was observed at power levels that we refer to as low here. ${ }^{3}$ The strong coupling anti-crossing, a SQD effect, could be seen even though the $Q$ was determined by an ensemble QD effect, namely the absorption of many partially saturated QDs. Both effects can occur simultaneously. For strong coupling, the QD must be situated near an anti-node, i.e., within an area of the slab of only $0.15 \mu \mathrm{m}^{2}$. The product of that area and the dot density of $400 \mu \mathrm{m}^{2}$ gives 60 QDs spread out over the $42.5 \mathrm{meV}$ linewidth. Therefore, there is a $0.7 \mathrm{meV}$ separation on average between QDs that could potentially couple strongly. However, if one computes ${ }^{3}$ the field distribution for our photoniccrystal-slab nanocavity, the field is nonzero over several $\mu \mathrm{m}^{2}$. This implies that there are many more QDs that can influence the linewidth, $Q$, and emission properties of the nanocavity, and they are spaced much closer together spectrally. The absorption of this background ensemble of QDs reduces the $Q$ at low power, and the gain of this ensemble leads to lasing. Emission from this ensemble enables the nanocavity peak to be seen even when the SQD is greatly detuned. It is intriguing that in principle an isolated SQD in our present nanocavity could exhibit lasing, but effects of the ensemble will have to be reduced considerably to see SQD lasing.

In summary, we have shown that the $Q$ of a GaAs photonic-crystal-slab nanocavity is now high enough that a single layer of high-density quantum dots introduces enough absorption to appreciably lower the $Q$, impeding the search for strong coupling, and enough gain for lasing.

\section{ACKNOWLEDGMENTS}

The Tucson group thanks DARPA, NSF AMOP and EPDT, and AFOSR; the Caltech group thanks the MURI Center for Photonic Quantum Information Systems (ARO/ ARDA), NSF-ECS-NIRT, and AFOSR; and the Texas group thanks NSF-ECS-NIRT for financial support.
${ }^{1}$ O. Painter, R. K. Lee, A. Scherer, A. Yariv, J. D. O'Brien, P. D. Dapkus, and I. Kim, Science 284, 1819 (1999).

${ }^{2}$ T. Yoshie, O. B. Shchekin, H. Chen, D. G. Deppe, and A. Scherer, Electron. Lett. 38, 967 (2002).

${ }^{3}$ T. Yoshie, A. Scherer, J. Hendrickson, G. Khitrova, H. M. Gibbs, G. Rupper, C. Ell, O. B. Shchekin, and D. G. Deppe, Nature (London) 432, 200 (2004).

${ }^{4}$ T. Yoshie, O. B. Shchekin, H. Chen, D. G. Deppe, and A. Scherer, IEICE Trans. Electron. E87-C, 300 (2004).

${ }^{5}$ Y. Akahane, T. Asano, B-S. Song, and S. Noda, Nature (London) 425, 944 (2003).

${ }^{6}$ D. Birkedal, J. Bloch, J. Shah, L. N. Pfeiffer, and K. West, Appl. Phys. Lett. 77, 2201 (2000).

${ }^{7}$ For example, for an interface fluctuation QD, J. R. Guest, T. H. Stievater, Xiaoqin Li, Jun Cheng, D. G. Steel, D. Gammon, D. S. Katzer, D. Park, C. Ell, A. Thränhardt, G. Khitrova, and H.
M. Gibbs, Phys. Rev. B 65, 241310(R) (2002).

${ }^{8}$ D. J. Mowbray and M. S. Skolnick, J. Phys. D 38, 2059 (2005).

${ }^{9}$ T. D. Happ, I. I. Tartakovskii, V. D. Kulakovskii, J-P. Reithmaier, M. Kamp, and A. Forchel, Phys. Rev. B 66, 041303(R) (2002).

${ }^{10}$ S. Mosor, J. Hendrickson, B. C. Richards, J. Sweet, G. Khitrova, H. M. Gibbs, T. Yoshie, A. Scherer, O. B. Shchekin, and D. G. Deppe, Appl. Phys. Lett.87, 141105 (2005).

${ }^{11}$ A. Badolato, K. Hennessy, M Atatüre, J. Dreiser, E. Hu, P. M. Petroff, and A. Imamoğlu, Science 308, 1158 (2005).

${ }^{12}$ R. Jin, D. Boggavarapu, M. Sargent III, P. Meystre, H. M. Gibbs, and G. Khitrova, Phys. Rev. A 49, 4038 (1994).

${ }^{13}$ I. Protsenko, P. Domokos, V. Lefèvre-Seguin, J. Hare, J. M. Raimond, and L. Davidovich, Phys. Rev. A 59, 1667 (1999).

${ }^{14}$ J. P. Reithmaier, G. Sękl, A. Löffler, C. Hofmann, S. Kuhn, S. Reitzenstein, L. V. Keldysh, V. D. Kulakovskii, T. L. Reinecke, and A. Forchel, Nature (London) 432, 197 (2004). 